Letter to the Editor

Human and Medical Genetics

\title{
The Leigh phenotype resulting from C12orf65 variants
}

\author{
Josef Finsterer ${ }^{1}[$ (] \\ ${ }^{1}$ Krankenanstalt Rudolfstiftung, Vienna, Austria.
}

Received: June 02, 2020; Accepted: July 17, 2020.

With interest we read the article by Perrone et al. (2020) about a 2 years old female with Leigh syndrome (LS) due to the homozygous $14 \mathrm{bp}$ frameshift deletion in C12orf65 encoding COXPD7. It was concluded that the mutated protein domain GGQ is responsible for a conservative peptidyl-hydrolase function and that loss of function mutations in this domain play a pathogenetic role for the development of LS (Perrone et al., 2020). We have the following comments and concerns.

We do not agree with the notion that LS is exclusively an early onset disease (Perrone et al., 2020). Though presenting with an onset $<2 \mathrm{y}$ of age in $80 \%$ of the cases (Hong et al., 2020), the remaining portion of LS patients has an onset $>2 \mathrm{y}$ of age (Hong et al., 2020).

We also do not agree with the statement that "brain changes are almost identical in all patients" with LS (Perrone et al., 2020). Though cerebral lesions are usually symmetric with regard to distribution, different structures may be affected. These include the caudate nucleus, the putamen, the globus pallidus, the thalamus, the brainstem, or the cerebellum (Finsterer, 2008). Though these miscellaneous presentations on imaging do not allow establishing close genotype-phenotype correlations, they represent to some extent the extensive genetic heterogeneity of the syndrome.

We should know if respiratory distress episodes were attributed to the brainstem lesion in the medulla oblongata, to affection of peripheral nerves affecting respiratory muscles, to myopathy of axial including respiratory muscles, or to lactic acidosis.

Though the C12orf65 deletion was present in the homozygous state and though DNA from the parents was extracted it is not mentioned if either parent carried the mutation in the heterozygous state, as could be expected. Sporadic occurrence of the variant is rather unlikely given the homozygous state.

Missing in the report is if lactate was measured in the cerebro-spinal fluid (CSF) or not. Neither did the patient undergo magnetic resonance spectroscopy (MRS), nor was a spinal tap carried out. Since cerebral lactic acidosis strongly determines the phenotype it is crucial to know if lactate was elevated in the CSF respectively the parenchyma or not.

Since some patients with LS may develop stroke-like episodes (SLEs) (Morin et al., 1999), we should know if the patient ever developed features of a SLE and if any of the cerebral MRIs ever showed a characteristic stroke-like lesion (SLL), the morphological equivalent of a SLE.

Since LS may go along with epilepsy and since epileptiform discharges were recorded on electroencephalography (EEG) (Perrone et al., 2020), we should know if the patient ever developed seizures or not and if treatment with anti-epileptic drugs (AEDs) was ever necessary or not.

Since cerebral MRI at age $2 \mathrm{y}$ and 9 months revealed bilaterally symmetric T2-hyperintense lesions in the substantia nigra, we should know if the patient ever developed Parkinsonism or a Parkinson-related disorder. Did the patient ever require anti-Parkinson drugs?

Overall, this interesting case has a number of shortcomings, which should be solved before the case is revaluated and final conclusions are drawn. Patients with LS are not only heterogeneous with regards to the genetic background but also with regard to the phenotype.

\section{References}

Finsterer J (2008) Leigh and Leigh-like syndrome in children and adults.Pediatr Neurol 39:223-235.

Hong CM, Na JH, Park S and Lee YM (2020) Clinical characteristics of early-onset and late-onset Leigh syndrome.Front Neurol 11:267.

Morin C, Dubé J, Robinson BH, Lacroix J, Michaud J, De Braekeleer M, Geoffroy G, Lortie A, Blanchette C, Lambert MA et al. (1999) Stroke-like episodes in autosomal recessive cytochrome oxidase deficiency.Ann Neurol 45:389-392.

Perrone E, Cavole TR, Oliveira MG, Virmond LDA, Silva MFB, Soares MFF, Iglesias SBO, Falconi A, Silva JS, Nakano V et al. (2020) Leigh syndrome in apatient with a novel C12orf65 pathogenic variant: Case report and literature review. Genet Mol Biol 43:e20180271. 


\title{
Author's reply to the comment by J. Finsterer
}

\author{
Eduardo Perrone ${ }^{1}$ (i) \\ ${ }^{1}$ Universidade Federal de São Paulo, Departamento de Genética Médica, São Paulo, SP, Brazil.
}

We really appreciated the interest and comments on our article (Perrone et al., 2020). Although we agree that different central nervous system structures can be affected in Leigh syndrome, and that there is considerable clinical, genetic, and biochemistry heterogeneity, when we pointed out that lesions were almost identical, we aimed to highlight symmetrical basal ganglia, brain stem, and cerebellum involvement, which is in line with previous descriptions (Finsterer, 2008).

Our neurological and clinical evaluation was limited, considering that the proband was under sedation and on mechanical ventilation. As the patient had brainstem signals in MRI, we believe that respiratory distress was mainly caused by brainstem involvement. However, it is not possible to rule out the influence of lactic acidosis, myopathy, or even peripheral nerve disorders. For your information, the CSF lactate level was $45.22 \mathrm{mg} / \mathrm{dL}$ (upper limit, $20 \mathrm{mg} / \mathrm{dL}$ ). We did not observe signals of stroke-like episodes (SLE) in our patient. Partial complex seizures were observed, partially controlled by carbamazepine, phenobarbital, and clobazam. MRI also revealed substantia nigra lesions, but we were not able to identify any Parkinsonism signs, considering all the above-mentioned limitations. Spectroscopy MRI would be informative, but it was not performed.

Regarding the genetic aspects, the consent was signed only by the mother, and we had access only to the proband's biological samples. Due to ethical and legal issues we could not contact the proband's father. However, parental consanguinity strongly suggests that the proband inherited identical pathogenic variants from both parents.

\section{References}

Finsterer J (2008) Leigh and Leigh-like syndrome in children and adults. Pediatr Neurol 39:223-235.

Perrone E, Cavole TR, Oliveira MG, Virmond LDA, Silva MFB, Soares MFF, Iglesias SBO, Falconi A, Silva JS, Nakano V et al. (2020) Leigh syndrome in apatient with a novel C12orf65 pathogenic variant: Case report and literature review. Genet Mol Biol 43:e20180271.

Associate Editor: Angela M. Vianna-Morgante

License information: This is an open-access article distributed under the terms of the Creative Commons Attribution License (type CC-BY), which permits unrestricted use, distribution and reproduction in any medium, provided the original article is properly cited. 